\title{
Multi-objective Optimal Dispatch in Power System Based On a Modified Multi-Objective Teaching-Learning Algorithm
}

\author{
Wang He, Yao Yapeng, Yue Jiawei and Guo Yueyue \\ School of Technology, Beijing Forestry University. \\ Beijing, China \\ wanghe0396@126.com
}

\begin{abstract}
With the increasing public awareness of environmental protection, limiting the emissions of polluting gas becomes a crucial objective in the power dispatch; power system dispatch converts from the original single objective economic dispatch to the multi-objective environmental/economic dispatch (EED), which is aimed at solving multi dimension, multi constrained and multi-constrained discontinuous problems of the multi-objective dispatching model. An improved multi-objective "teaching" and "learning" optimization algorithm (MMTLA) is got and applied to solve the model in this paper. In terms of the problem that losing the diversity of particles easily and early in the original teaching and learning algorithm leads to fall into local optimization problem, firstly, the search performance of the algorithm can be improved through the introduction of quasi-opposition-based learning and self-learning mutation. Secondly, obtain global Pareto optimal solution set and a true Pareto front by introducing alternative set and macro perturbation operations, and then select the best compromise solution based on fuzzy theory. Finally, several optimization trials of the proposed algorithm are carried out on the IEEE 30-bus test system. The simulation results demonstrate superiority of the proposed approach and confirm its potential to solve the multiobjective EED problem.
\end{abstract}

Keywords: environmental/economic dispatch; modified multi-objective teaching-learning algorithm; power system

\section{Introduction}

The classical power system economic dispatch only pursues the minimum cost of power generation of a single objective (economic dispatch) when meeting the operating constraints [1]. With the increasing public awareness of the environmental pollution caused by fossil fuel fired thermal power plants, this power dispatch is supposed to build the multi-objective dispatching model considering the fuel cost and emission of pollutants simultaneously on the basis of the single objective dispatch [2].

Power system optimal dispatch is a multi-objective optimization problem with high dimension, nonlinearity and multi constraints. The traditional multi-objective optimization method is to convert the multi-objective optimization problem into a single objective optimization problem with coefficients in a weighted way, and then use the linear or nonlinear optimization method to solve the problem. With the development of computer and the improvement of artificial intelligence technology, intelligent algorithm is widely used in power system optimization dispatching problem, the quantum computation and genetic algorithm are combined to form a quantum genetic algorithm, which is used to solve the economic dispatch problem (as suggested in [3]), genetic algorithm and bacterial foraging algorithm for solving the economic dispatch problem in [4]. As pointed out in [5], it is a comprehensive improvement of the basic GA algorithm, which is based on the operation of the chaos operator and the quantum operator ; what's more, it has been applied into the 
environmental economic dispatch of micro grid and the result demonstrates pretty well. Combined with the fuzzy theory, the fuzzy theory and PSO algorithm are effectively integrated to form a new hybrid algorithm, which is used to optimize the dynamic economic dispatch. The research shows that the algorithm has better convergence performance and strong search ability in this paper [6]. The dynamic economic dispatch problem with valve point effect is solved by combining the chaos operator and the evolutionary algorithm in [7]. Hybrid interior point method and DE forms a new hybrid evolutionary algorithm in [8]. The optimization problem of multi-objective scheduling can bring good social and economic benefits, so researchers have been active in the exploration of power system scheduling model based on the new algorithm. Multi-objective dispatching problem of power system is solved by using multiple objective fuzzy dominant bacteria foraging algorithm in [9]. A kind of imperialist competitive algorithm is adopted to optimize the dynamic economic dispatch model in [10]. Teaching and learning algorithm is a new swarm intelligence optimization algorithm proposed by Rao et al in 2010 [11], which simulates the process of teaching and learning of the teachers to the learners. The purpose is to improve the learners' learning performance by the teacher's "teaching" and "learning". Compared with other intelligent algorithms, the biggest advantage of the teaching and learning algorithm is that the parameters are few. The real algorithm does not need any set of parameters, which greatly reduces the time and the robustness of the algorithm. Moreover, the algorithm has a strong convergence speed. Teaching and learning algorithm from the proposed to the present time, has attracted many researchers' interests, and has got a very good application [11-13].

This paper explores the Pareto theory and improved teaching and learning algorithm fusion, to solve the multi-objective dispatching problem of power system, the problem that the original teaching and learning algorithm is easy to premature loss of diversity of particles and fall into the local optimum problem can be improved by introducing self-learning mechanism of variation and the reverse learning operation to improve the algorithm search performance, by introducing alternative set history obtained Pareto optimal solution set and the global Pareto optimal solution set to improve the algorithm's global search ability and through the introduction of macro disturbance operation to find the true Pareto front; finally, select the best compromise solution using fuzzy theory. At last, the algorithm is verified on the IEEE6 30 bus system, and the comparison of other algorithms shows that the algorithm is advanced and robust.

\section{Mathematical Model}

The multi-objective dispatching problem in power system is to obtain the optimal results of the economic dispatch objective and the environmental dispatch objective when meeting the requirements of the power supply and demand balance and the output limit of the power system. The result is the optimal value of the fuel cost and pollution gas emissions. The mathematical description of multi-objective scheduling problem for power system is formulated as follows.

\subsection{Objective Functions}

\subsubsection{Economic Dispatch}

Economic dispatch is aimed at minimizing the cost of fuel for scheduling objectives. The fuel cost curve for each generating unit is usually represented by a function of two variables; its total fuel cost $F\left(P_{G}\right)$ can be represented as:

$$
F\left(P_{G}\right)=\sum_{i=1}^{N}\left(a_{i}+b_{i} P_{G i}+c_{i} P_{G i}^{2}\right)
$$

Where $N$ is total number of thermal power units; $a_{i}, b_{i}$ and $c_{i}$ are the fuel cost coefficients of the $i$ th generator; $P_{G i}$ is the real power output of the $i$ th generator. 


\subsubsection{Environmental Dispatch}

Environmental scheduling is aimed at minimizing the amount of the atmospheric pollutants emission for scheduling objectives. In the process of generating electricity, the thermal power units emit many kinds of atmospheric pollutants, and each emission of atmospheric pollutants can be established as a function relationship with the active power unit respectively, but for the convenience of calculation, a model of the comprehensive atmospheric pollutants emission is applied in this paper, and the total emission of the atmospheric pollutants can be presented as [10].

$$
E\left(P_{G}\right)=\sum_{i=1}^{N}\left[10^{-2}\left(\alpha_{i}+\beta_{i} P_{G i}+\gamma_{i} P_{G i}^{2}\right)+\zeta_{i} \exp \left(\lambda_{i} P_{G i}\right)\right]
$$

Where $\alpha_{i}, \beta_{i}, \gamma_{i}, \zeta_{i}$ and $\lambda_{i}$ are the emission coefficients of the $i$ th generator.

\subsection{Constraints}

1) Power balance constraint

$$
\sum_{i=1}^{N} P_{G i}-P_{D}-P_{l}=0
$$

Where $P_{D}$ is the total system load demand, $P_{l}$ is the real power loss in transmission lines.

2) Generation capacity constraint

$$
P_{G i \min } \leq P_{G i} \leq P_{G i \max }
$$

Where $P_{G i m i n}$ and $P_{G i \max }$ are the minimum active power and maximum active power output of the $i$ th generator respectively.

3) System spinning reserve constraint

$$
\sum_{i=1}^{N} P_{G i \max }-P_{D}-P_{l} \geq P_{S R}
$$

Where $P_{S R}$ is the system of the reserve capacity, and it is 5 percent of the total load generally.

\subsection{Mathematical Model}

Aggregating the above objectives and constraints, the EED optimization problem can be described as:

$$
\left\{\begin{array}{l}
\min Y(X)=\min [F(X), E(X)] \\
\text { s.t. } \\
\quad h_{i}(X)=0, i=1,2, \ldots, L \\
g_{j}(X) \geq 0, j=1,2, \ldots, K
\end{array}\right.
$$

Where $X=P_{G}$ is the solution vector of optimization problem; $h$ and $g$ are the equality and inequality constraints in the model, respectively; $L$ and $K$ is the number of equality and inequality constraints.

\section{Improve Multi-Objective Teaching and Learning Algorithm}

\subsection{Optimization and Improvement of the Classical Teaching and Learning Algorithm}

Teaching and learning algorithm can solve the relevant problems by simulating the teaching and learning process of the teachers and learners [11-13].The steps of teaching and learning algorithm are as follows:

Step 1 : Initialize the parameters of the learners. Each student $X^{i}=\left(x_{1}^{i}, x_{2}^{i}, \cdots, x_{d}^{i}\right)(i=1,2, \cdots, N P)$ generated randomly on the search space. 
Step 2: Teaching. In the "teaching" stage of teaching and learning algorithm, each student $X^{i}$ in the class learns according to the difference between $X_{\text {teacher }}$ and the average value mean .

$$
\left\{\begin{array}{l}
X_{\text {new }}^{i}=X_{\text {old }}^{i}+\text { difference } \\
\text { difference }=r_{i} \times\left(X_{\text {teacher }}-T F_{i} \times \text { mean }\right)
\end{array}\right.
$$

Where $X_{\text {new }}^{i}$ and $X_{\text {old }}^{i}$ mean the antecedent and subsequent the value of the ith learner; mean is the average value of all the learners for the iteration; mean $=\frac{1}{N P} \sum_{i=1}^{N P} X^{i}, N P$ means the number of all the learners; $T F^{i}$ is the learning factor of the $i$ th learner; $T F^{i}=\operatorname{round}[1+\operatorname{rand}(0,1)]$, Learning step can be represented as $r_{i}=\operatorname{rand}(0,1)$.

Step 3: Studying. For each student $X^{i}$, selecting a learning object $X^{j}(j=1,2 \ldots, N P, j \neq i)$ randomly in the class and $X^{i}$ learns to adjust with the analysis of the differences between themselves and the student $X^{j}$. The method to improve learning is similar to the differential mutation operator in differential algorithm, but the difference is that the learning step $r_{i}=\operatorname{rand}(0,1)$ in the teaching and learning algorithm is different for each student.

$$
X_{\text {new }}^{i}=\left\{\begin{array}{ll}
X_{\text {old }}^{i}+r_{i} \times\left(X^{i}-X^{j}\right) & f\left(X^{j}\right)<f\left(X^{i}\right) \\
X_{\text {old }}^{i}+r_{i} \times\left(X^{j}-X^{i}\right) & f\left(X^{i}\right)<f\left(X^{j}\right)
\end{array}\right\}
$$

Where $f\left(X^{i}\right)$ and $f\left(X^{j}\right)$ are the fitness values of the $i$ th and $j$ th learners, respectively.

Step 4: Update operation

$$
\begin{aligned}
& \text { if } \quad f\left(X_{\text {new }}^{i}\right)>f\left(X_{\text {old }}^{i}\right) \\
& X_{\text {new }}^{i}=X_{\text {old }}^{i} \\
& \text { end if }
\end{aligned}
$$

Classical TLA algorithm has a fast search speed, but it is apt to lose the diversity of the learner and suffer the problem of being trapped in local optima. In this paper, the approximate inverse learning and self - learning mutation operation are introduced to improve the classical teaching and learning algorithm, specific operations are as follows:

a) Approximate inverse learning

The related research shows that the approximate inverse learning is more likely to obtain the optimal solution. Therefore, dynamic quasiopposition-based learning is adopted to improve the convergence speed of the intelligent algorithm.

The approximate dynamic quasiopposition-based learning:

$$
X_{i, j}^{t, *}=\operatorname{rand}^{*}\left(\min \left(X_{i, j}^{t}\right)+\max \left(X_{i, j}^{t}\right)\right)-X_{i, j}^{t}
$$

Where $X_{i, j}^{t}$ means the current population of the $i$ th dimension of the $j$ th learner; $\min \left(X_{i, j}^{t}\right)$ and $\max \left(X_{i, j}^{t}\right)$ are the upper and lower bounds of the $j$ th dimension of the $i$ th learner, respectively.

b) Self - learning mutation operation

The main learning styles of the learners are to learn from the teacher and the learners, but there is no specific mechanism for the basic teaching and learning. Therefore, a self - learning mutation mechanism is proposed in this paper and it can be defined as:

$$
X_{\text {new }}^{t, *}=X_{\text {old }}^{j}+\operatorname{rand}(0,1) \times\left(\max \left(X_{i, j}^{t}\right)-\min \left(X_{i, j}^{t}\right)\right)
$$


Where $\min \left(X_{i, j}^{t}\right)$ and $\max \left(X_{i, j}^{t}\right)$ are the upper and lower bounds of the $i$ th learner with respect to the $j$ th dimension, respectively.

\subsection{Improve the Process of Multi-Object Teaching and Learning Optimization Algorithm}

Alternative sets and macro disturbance are introduced to improve the diversity and approximation of the Pareto optimal solution set, and to form a new type of multi object teaching and learning algorithm on the basis of the modified multi-objective teaching learning algorithm.The generic steps of the proposed algorithm are as follows:

a) Initialize the algorithm. 1) Initialize population, including population size, problem dimension, and evaluation of learners; 2) initialize alternate selection set and Pareto optimal solution set.

b) Choose the best individual in the population as the teacher.

c) According to the Eq.(8) and (9), Form a group of new learners

d) Restrain the new learners to evaluate other learners, and update the learners and the teacher.

e) Update alternate selection set and Pareto optimal solution set according to the domination definition. Store non-dominated solution to the standby selection set, and the feasible solution is stored to the Pareto optimal solution set; Check the number of individuals in the standby selection set and Pareto optimal solution set, if it is greater than the set value, remove the redundant individual based on the nearest neighbor distance of the $\mathrm{K}$ until the individual number of the standby selection is satisfied.

The nearest neighbor distance of the $\mathrm{K}$ :

$$
d_{i, j}=\sqrt{\sum_{k=1}^{N_{o b j}}\left(\frac{f_{k}^{i}-f_{k}^{j}}{f_{k}^{\max }-f_{k}^{\min }}\right)^{2}}
$$

Where $N_{o b j}$ is the number of objective functions; $f_{k}^{i}$ and $f_{k}^{j}$ are the $k$ th dimension target function values of the $i$ th and $j$ th learners, respectively; $f_{k}^{\max }$ and $f_{k}^{\min }$ are the maximum and minimum values of the $k$ th dimension.

f) Make macro disturbance to the Pareto optimal set, and update the Pareto optimal solution set according to the control definition.

The corresponding target region of Pareto optimal solution set gradually converges to the true Pareto-optimal front. At the moment, At this point, the Pareto optimal solution set is close to the true Pareto-optimal front. If macro disturbance can be applied into the Pareto optimal solution to form new learners, it will make the learner more competent to obtain the true Pareto-optimal front.

Macro disturbance operation: supposed that a Pareto-optimal solution is $X=\left[x_{1}, x_{2}, \ldots, x_{n}\right]$, the macro perturbation equation is shown as follows:

$$
x_{i}=x_{i}+p x_{i}\left(T_{\max }-t\right) / T_{\max }
$$

Where $p$ means initial mutation probability and its value is 0.02 in the paper; $t$ and $T_{\max }$ are the current iteration number and maximum number of iterations, respectively. With the increase of the number of iterations, the Pareto optimal solution set is more close to the real optimal frontier, and the variation probability will be smaller.

h) Updated global optimal solution

i) To determine whether the maximum number of iterations is reached, then stop the iteration; otherwise, the iteration number is 1 , and return to step b) and continue iterating.

j) Output Pareto optimal front, choose the best compromise solution according to the decision maker.

In this paper, the fuzzy membership function method is used to evaluate the satisfaction of the Pareto optimal solution, the satisfaction of the $i$ th Pareto optimal solution with respect to the $k$ th dimension objective function is: 


$$
\mu_{i}^{k}=\left\{\begin{array}{lc}
1, & f_{k}^{i} \leq f_{k}^{\min } \\
\frac{f_{k}^{\max }-f_{k}^{i}}{f_{k}^{\max }-f_{k}^{\min }}, & f_{k}^{\min } \leq f_{k}^{i} \leq f_{k}^{\max } \\
0, & f_{k}^{i} \geq f_{k}^{\max }
\end{array}\right.
$$

The satisfaction of each Pareto optimal solution is shown in the Eq. (14), and choose the most optimal solution as the compromise solution.

$$
\mu_{i}=\sum_{k=1}^{N_{o b j}} \mu_{i}^{k}
$$

\section{Simulation Test}

\subsection{Single Objective Optimization}

In this paper, the proposed algorithm is carried out on the IEEE 30-bus test system, and the relevant parameters of the system are found in [16]. The total load of the system is $283.4 \mathrm{MW}$ and the upper and lower bounds of the output of the generator sets, the fuel cost coefficient and the pollutant discharge coefficient are listed in Table 1.

\begin{tabular}{|c|c|c|c|c|c|}
\hline $\begin{array}{l}\text { Unit } \\
\text { num } \\
\text { ber }\end{array}$ & $\begin{array}{l}a l \\
\$\end{array}$ & $\begin{array}{c}b / \\
(\$ /(\mathrm{MW} \\
\cdot \mathrm{h}))\end{array}$ & $\begin{array}{c}c l \\
\left(\$ /\left(\mathrm{MW}^{2}\right.\right. \\
\cdot \mathrm{h}))\end{array}$ & $\begin{array}{l}P_{\min } I \\
\text { MW }\end{array}$ & $\begin{array}{l}P_{\max /} \\
\text { MW }\end{array}$ \\
\hline$G_{1}$ & 10 & 2.0 & 0.010 & 5 & 150 \\
\hline$G_{2}$ & 10 & 1.5 & 0.012 & 5 & 150 \\
\hline$G_{3}$ & 20 & 1.8 & 0.004 & 5 & 150 \\
\hline$G_{4}$ & 10 & 1.0 & 0.006 & 5 & 150 \\
\hline$G_{5}$ & 20 & 1.8 & 0.004 & 5 & 150 \\
\hline$G_{6}$ & 10 & 1.5 & 0.010 & 5 & 150 \\
\hline $\begin{array}{l}\text { Unit } \\
\text { num } \\
\text { ber }\end{array}$ & $\begin{array}{l}\alpha /(\mathrm{t} / \\
\mathrm{h})\end{array}$ & $\begin{array}{c}\beta / \\
(\mathrm{t} /( \\
\mathrm{MW} \cdot \mathrm{h}) \\
)\end{array}$ & $\begin{array}{c}\gamma / \\
\left(\mathrm{t} /\left(\mathrm{MW}^{2} .\right.\right. \\
\mathrm{h}))\end{array}$ & $\begin{array}{c}\zeta /(\mathrm{t} / \mathrm{h} \\
)\end{array}$ & $\begin{array}{c}\lambda / \\
\mathrm{MW}^{-1}\end{array}$ \\
\hline$G_{1}$ & $\begin{array}{c}4.09 \\
1\end{array}$ & $\begin{array}{c}5.554 \times \\
10^{-2}\end{array}$ & $\begin{array}{c}6.490 \times 1 \\
0^{-4}\end{array}$ & $\begin{array}{c}2.0 \times 1 \\
0^{-4}\end{array}$ & $\begin{array}{c}0.0285 \\
7\end{array}$ \\
\hline$G_{2}$ & $\begin{array}{c}2.54 \\
3\end{array}$ & $\begin{array}{c}- \\
6.047 x \\
10^{-2}\end{array}$ & $\begin{array}{c}5.638 \times 1 \\
0^{-4}\end{array}$ & $\begin{array}{c}5.0 \times 1 \\
0^{-4}\end{array}$ & $\begin{array}{c}0.0333 \\
3\end{array}$ \\
\hline$G_{3}$ & $\begin{array}{c}4.25 \\
8\end{array}$ & $\begin{array}{c}- \\
5.094 \times \\
10^{-2}\end{array}$ & $\begin{array}{c}4.586 \times 1 \\
0^{-4}\end{array}$ & $\begin{array}{c}1.0 \times 1 \\
0^{-6}\end{array}$ & $\begin{array}{c}0.0800 \\
0\end{array}$ \\
\hline$G_{4}$ & $\begin{array}{c}5.32 \\
6\end{array}$ & $\begin{array}{c}- \\
3.550 \times \\
10^{-2}\end{array}$ & $\begin{array}{c}3.380 \times 1 \\
0^{-4}\end{array}$ & $\begin{array}{c}2.0 \times 1 \\
0^{-3}\end{array}$ & $\begin{array}{c}0.0200 \\
0\end{array}$ \\
\hline$G_{5}$ & $\begin{array}{c}4.25 \\
8\end{array}$ & $\begin{array}{c}- \\
5.094 \times \\
10^{-2}\end{array}$ & $\begin{array}{c}4.586 \times 1 \\
0^{-4}\end{array}$ & $\begin{array}{c}1.0 \times 1 \\
0^{-6}\end{array}$ & $\begin{array}{c}0.0800 \\
0\end{array}$ \\
\hline$G_{6}$ & $\begin{array}{c}6.13 \\
1\end{array}$ & $\begin{array}{c}- \\
5.555 x \\
10^{-2}\end{array}$ & $\begin{array}{c}5.151 \times 1 \\
0^{-4}\end{array}$ & $\begin{array}{c}1.0 \times 1 \\
0^{-5}\end{array}$ & $\begin{array}{c}0.0666 \\
7\end{array}$ \\
\hline
\end{tabular}

Table 1. The Data of the IEEE 30-Bus Test System 
In order to show the effectiveness of the proposed algorithm in this paper, firstly, a single objective dispatching optimization is carried out and compared with other results of the literature.

In order to obtain the two boundary solutions of the optimal frontier of Pareto, the solution of the minimum value of the fuel cost and the pollution gas emissions, and verify whether the improved algorithm has a good distribution of the solution on the POF or not, the first step is to operate in economic and environment dispatch using the single objective teaching and learning algorithm. Particle number is forty and the maximum iteration number is 100 in the algorithm. Optimal solution can be obtained and listed in Table 2 and 3 with the calculation of the proposed algorithm. Figure 3 and Figure 4 represent the convergence curve of the economic dispatch and the environment dispatch, and it can be seen from the figures above, the algorithm has a better convergence performance.

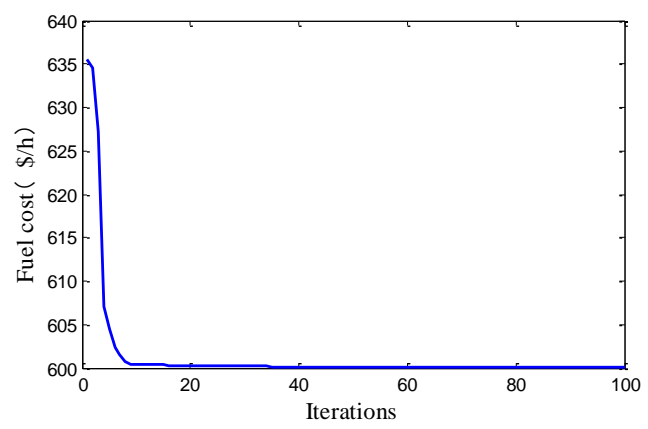

Figure 2. Convergencecurve for Economic Dispatch

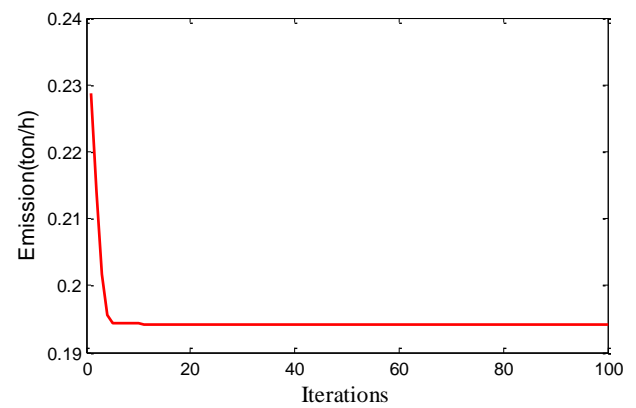

Figure 3. Convergencecurve for Environmental Dispatch

Table.2. The Results For Economic Dispatch and Environmental Dispatch

\begin{tabular}{|c|c|c|c|c|c|c|c|c|c|}
\hline \multirow{5}{*}{ 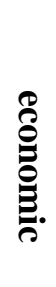 } & algorithm & $G_{1}$ & $G_{2}$ & $G_{3}$ & $G_{4}$ & $G_{5}$ & $G_{6}$ & Fuel cost $/(\$ / \mathrm{h}$ & $\begin{array}{c}\text { pollutant/(t/ } \\
\mathrm{h})\end{array}$ \\
\hline & MMTLA & 10.9732 & 29.9757 & 52.4302 & 101.6185 & 52.4250 & 35.9774 & 600.1114 & 0.2221 \\
\hline & $\mathrm{NSGA}^{[17]}$ & 15.67 & 28.70 & 46.71 & 104.67 & 50.37 & 37.29 & 600.572 & 0.22282 \\
\hline & FCPSO $^{[16]}$ & 10.70 & 28.97 & 52.5 & 101.5 & 53.00 & 36.73 & 600.132 & 0.2226 \\
\hline & MODEPSO $^{1+13}$ & 10.78 & 30.4 & 52.37 & 101.47 & 52.23 & 36.16 & 600.115 & 0.22201 \\
\hline & SPEA $^{[16]}$ & 10.62 & 28.97 & 52.89 & 100.25 & 54.02 & 36.64 & 600.150 & 0.22151 \\
\hline 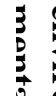 & algorithm & $G_{1}$ & $G_{2}$ & $G_{3}$ & $G_{4}$ & $G_{5}$ & $G_{6}$ & Fuel $\cos /(\$ / \mathrm{h})$ & $\begin{array}{c}\text { pollutant/(t/ } \\
\mathrm{h})\end{array}$ \\
\hline
\end{tabular}




\begin{tabular}{ccccccccc}
\hline NSGA $^{[17]}$ & 43.94 & 45.11 & 51.05 & 38.71 & 55.53 & 49.05 & 639.209 & 0.194356 \\
FCPSO $^{[16]}$ & 40.97 & 45.5 & 53.63 & 38.42 & 53.48 & 51.40 & 638.358 & 0.194207 \\
MODEPSO $^{[15}$ & 40.61 & 45.81 & 54.08 & 38.22 & 53.76 & 50.91 & 638.27 & 0.194203 \\
SPEA $^{[16]}$ & 41.16 & 45.32 & 53.29 & 38.32 & 53.83 & 51.48 & 638.507 & 0.194210 \\
\hline
\end{tabular}

As seen from the Figures 2 and 3 and the Table 2, the brief conclusion can be summarized as follows:

a)The proposed algorithm has a fast convergence rate and can quickly converge to the optimal solution which has a better convergence performance from the figures.

b) Compared with those reported algorithms such as NSGA, SPEA,FCPSO and MODEPSO, the proposed algorithm has an excellent performance in searching; as seen from the economic dispatch in the Table 2, the result of the proposed is better than other algorithms; the result of the environmental dispatch is similar to the MODEPSO algorithm, but it is more convenient to obtain the correct solution.

\subsection{Multi-Objective Scheduling Optimization}

In multi-objective scheduling optimization, the number of each generation particles is set to 40 , the maximum number of iterations 100 , and the number of solutions on the Pareto optimal frontier is set to 30 in the improved teaching and learning algorithm. The results of POF Using the MMTLA in Figure 4. Table 3 depicts the optimal solution set for the optimal Pareto solution.

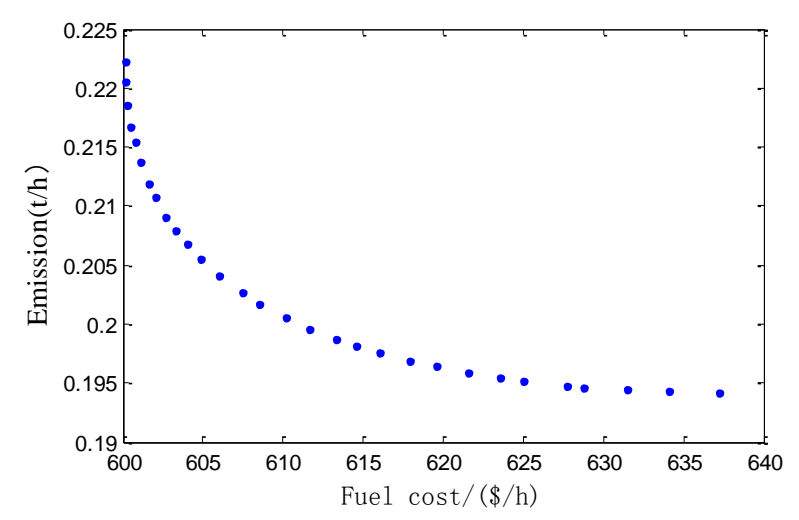

Figure 4. POF Using the MMTLA

Table 3. Boundary Solution of POF

\begin{tabular}{cccc}
\hline Unit number & $\begin{array}{c}\text { Optimal } \\
\text { economic }\end{array}$ & $\begin{array}{c}\text { Optimal } \\
\text { environment }\end{array}$ & $\begin{array}{c}\text { Compromise } \\
\text { optimal } \\
\text { solution }\end{array}$ \\
\hline$G_{1}$ & 11.0099 & 27.0007 & 26.0184 \\
$G_{2}$ & 30.0170 & 38.2604 & 40.1608 \\
$G_{3}$ & 52.4191 & 53.0876 & 56.3307 \\
$G_{4}$ & 101.6271 & 72.1794 & 62.6697 \\
$G_{5}$ & 52.4347 & 56.6009 & 52.0015 \\
$G_{6}$ & 35.8922 & 36.2711 & 46.2188 \\
\hline Fuel cost $(\$ / \mathrm{h})$ & 600.1471 & 639.3214 & 607.0792 \\
Pollution & 0.2209 & 0.1942 & 0.2030 \\
gas/(t/h) & & &
\end{tabular}


Comparing the results of Table 2 with Table 3, we can see that the difference between Boundary solution of POF and the The results for signal goal dispatch is very small, which shows that the multi-objective algorithm proposed in this paper can find a better boundary solution in different conditions and the algorithm has a better robustness.

\section{Conclusion}

In this paper, a new multi-objective teaching - learning algorithm is proposed to solve multi-objective dispatching problem in the power system. Firstly, the search performance of the algorithm can be improved through the introduction of quasi-opposition-based learning and self-learning mutation. Secondly, obtain global Pareto optimal solution set and a true Pareto front by introducing alternative set and macro perturbation operations, and then select the best compromise solution based on fuzzy theory. Finally, several optimization trials of the proposed algorithm are carried out on the IEEE 30-bus test system. Therefore, we can obtain three points of the paper as follows:

(a)The proposed algorithm has good stability and convergence. It can find the global optimal solution under different initial solutions and the convergence rate is fast;

(b) With the proposed algorithm, the improved algorithm can get the POF, which has a uniform and wide distribution, and the algorithm is simple and easy to operate;

(c) The algorithm can not only be used to solve the multi-objective scheduling problem of power system, but also can provide technical support for other areas of large-scale, multidimensional, multi constrained non-continuous optimization problems.

\section{Acknowledgements}

This project is supported by the Fundamental Research Funds for the Central University (No.BLX2014-05, NO.2015ZCQ- GX-04).

\section{References}

[1] J. B. Park, K. S. Lee and J. R. Shin, "A particle swarm optimization for economic dispatch with nonsmooth cost functions", IEEE Transactions on Power Systems, vol. 20, no. 1, (2005), pp. 34-42.

[2] T. Niknam and H. Doagou-Mojarrad, "Multi-objective economic/emission dispatch by multi-objective $\Theta-$ particle swarm optimization”, Transmission \& Distribution, vol. 6, no. 5, (2012), pp. 363-377.

[3] J. C. Lee, W. M. Lin and G. C. Liao, "Quantum genetic algorithm for dynamic economic dispatch with valve-point effects and including wind power system", International Journal of Electrical Power \& Energy Systems, vol. 33, no. 2, (2011), pp. 189-197.

[4] X. S. Yang, S. S. Sadat Hosseini and A. H. Gandomi, "Firefly algorithm for solving non-convex economic dispatch problems with valve loading effect", Applied Soft Computing, vol. 12, no. 3, (2012), pp. 11801186.

[5] G. C. Liao, "Solve environmental economic dispatch of Smart MicroGrid containing distributed generation system-Using chaotic quantum genetic algorithm", International Journal of Electrical Power \& Energy Systems, vol. 43, no. 1, (2012), pp. 779-787.

[6] T. Niknam, "A new fuzzy adaptive hybrid particle swarm optimization algorithm for non-linear, non-smooth and non-convex economic dispatch problem", Applied Energy, vol. 87, no. 1, (2010), pp. 327-339.

[7] Y. Lu, J. Zhou and H. Qin, "Chaotic differential evolution methods for dynamic economic dispatch with valve-point effects”, Engineering Applications of Artificial Intelligence, vol. 24, no. 2, (2011), pp. 378-387.

[8] N. Duvvuru and K. S. Swarup, “A hybrid interior point assisted differential evolution algorithm for economic dispatch", Power Systems, IEEE Transactions on, vol. 26, no. 2, (2011), pp. 541-549.

[9] B. K. Panigrahi, V. RavikumarPandi and S. Das, "Multiobjective fuzzy dominance based bacterial foraging algorithm to solve economic emission dispatch problem", Energy, vol. 35, no. 12, (2010), pp. 4761-4770.

[10] B. Mohammadi-ivatloo, A. Rabiee and A. Soroudi, "Imperialist competitive algorithm for solving nonconvex dynamic economic power dispatch”, Energy, vol. 44, no. 1, (2012), pp. 228-240.

[11] R. V. Rao, V. J. Savsani and D. P. Vakharia, "Teaching-learning-based optimization: a novel method for constrained mechanical design optimization problems", Computer Aaided Design, vol. 43, no. 3, (2011), pp. 303-315.

[12] T. Niknam and R. Azizipanah-Abarghooee Aghaeij, “A new modified teaching-learing algorithm for reserve constrained dynamic economic dispatch, IEEE Transactions on Power Systems, vol. 13, no. 3, (2013), pp. 749-763. 
[13] R. V. Rao, V. J. Savsani and J. Balic, "Teaching learning-based optimization algorithm for unconstrained and constrained real parameter optimization problems", Engineering Optimization, vol. 44, no. 2, (2012), pp. 1447-1462.

[14] P. Chunhua and S. Huijuan, "Multi-objective optimization power dispatch based on non-dominated sorting differential evolution", Proceedings of the CSEE, vol. 29, no. 34, (2009), pp. 71-76

[15] K. P. Wong and C. C. Fung, "Simulated annealing based economic dispatch algorithm", Generation, Transmission and Distribution, IEE Proceedings, vol. 140, no. 6, (1993), pp. 509-515.

[16] D.-W. Gong, Y. Zhang and C.-L. Qi, "Environmental /economic power dispatch using a hybrid multiobjective optimization algorithm", Electrical Power and Energy Systems, vol. 32, (2010), pp. 607-614.

[17] S. Rahnamayan, H. R. Tizhoosh and S. M. Ma, "Quasi oppositional differential evolution", // Proceeding of IEEE Congress on Evolution Computation, CEC 2007,September 25-28, (2007), pp. 2229-2236.

[18] P. K. Roy and D. Mandal, "Quasi-oppositional biogeography-based optimization for multi-objective optimal power flow”, Electric Power Components and Systems, vol. 40, no. 2, (2011), pp. 236-256.

\section{Authors}
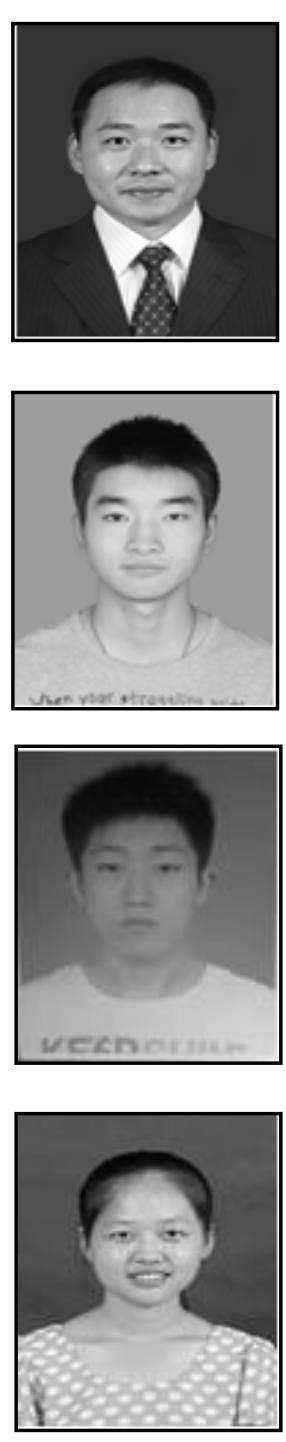

Wang He, he is currently a lecturer with the School of Technology, Beijing Forestry University, His research interests include Wind power forecasting, micro grid energy management.

Yao Yapeng, he was born in Zhengyang, Henan province, China. As a senior student, he majors in electrical engineering and automation in School of Technology, Beijing Forestry University from 2012.

Yue Jiawei, he was born in Baoding, Hebei province, China. As a bachelor candidate, he majors in electrical engineering and automation in School of technology, Beijing Forestry University from 2013.

Guo Yueyue, she was born in Pingdingshan, Henan province, China. As a bachelor candidate, she majors in electrical engineering and the automation specialty of Beijing Forestry University from 2013 . 